\title{
Succinate Dehydrogenase [Ubiquinone] Flavoprotein Subunit, Mitochondrial
}

National Cancer Institute

\section{Source}

National Cancer Institute. Succinate Dehydrogenase rUbiquinone] Flavoprotein Subunit, Mitochondrial. NCI Thesaurus. Code C103875.

Succinate dehydrogenase [ubiquinone] flavoprotein subunit, mitochondrial (664 aa, 73 $\mathrm{kDa}$ ) is encoded by the human SDHA gene. This protein is involved in the mitochondrial electron transport chain. 